\title{
On the Möbius Function of Permutations with One Descent
}

\author{
Jason P. Smith \\ Department of Computer and Information Sciences \\ University of Strathclyde \\ Glasgow, U.K. \\ jason.p.smith@strath.ac.uk
}

Submitted: July 8, 2013; Accepted: Apr 3, 2014; Published: Apr 16, 2014

Mathematics Subject Classification: 05 A05

\begin{abstract}
The set of all permutations, ordered by pattern containment, is a poset. We give a formula for the Möbius function of intervals $[1, \pi]$ in this poset, for any permutation $\pi$ with at most one descent. We compute the Möbius function as a function of the number and positions of pairs of consecutive letters in $\pi$ that are consecutive in value. As a result of this we show that the Möbius function is unbounded on the poset of all permutations. We show that the Möbius function is zero on any interval $[1, \pi]$ where $\pi$ has a triple of consecutive letters whose values are consecutive and monotone. We also conjecture values of the Möbius function on some other intervals of permutations with at most one descent.
\end{abstract}

\section{Introduction}

Let $\sigma$ and $\pi$ be permutations of positive integers. We define an occurrence of $\sigma$ as a pattern in $\pi$ to be a subsequence of $\pi$ with the same relative order of size as the letters in $\sigma$. For example, if $\sigma=213$ and $\pi=23514$ then there are two occurrences of $\sigma$ in $\pi$ as the subsequences 214 and 314. A permutation $\pi$ is said to avoid a pattern $\sigma$ if there are no occurrences of $\sigma$ in $\pi$. The set of all permutations forms a poset $\mathcal{P}$, with a partial ordering defined as $\sigma \leqslant \pi$ if $\sigma$ occurs as a pattern in $\pi$. An interval $[\sigma, \pi]$ in $\mathcal{P}$ is a subposet consisting of all permutations $z \in \mathcal{P}$ with $\sigma \leqslant z \leqslant \pi$. The Möbius function is defined recursively as follows: $\mu(\sigma, \lambda)=0$ if $\sigma \nless \lambda, \mu(\sigma, \sigma)=1$ for all $\sigma$ and for $\sigma<\lambda$ :

$$
\mu(\sigma, \lambda)=-\sum_{\sigma \leqslant z<\lambda} \mu(\sigma, z) .
$$

We frequently use the term Möbius value of a permutation $\lambda$ to refer to $\mu(1, \lambda)$ and we refer to permutations with a nonzero Möbius value as nonzero permutations. A descent 
in a permutation $\pi=\pi_{1} \pi_{2} \ldots \pi_{n}$ is a decrease in the value of consecutive letters, that is, a descent at position $i$ is where $\pi_{i}>\pi_{i+1}$.

Formulas for the Möbius function in this poset in certain special cases have been proved. Almost all such results so far are on permutations constructed using direct sums, where the direct sum of two permutations $\alpha$ and $\beta$, denoted $\alpha \oplus \beta$, is the concatenation of $\alpha$ with $\beta^{\prime}$, where $\beta^{\prime}$ is the permutation $\beta$ with each letter increased in value by the length of $\alpha$. For example $213 \oplus 2413=2135746$. The first such result was by Sagan and Vatter in [SV06], where they give a formula for the Möbius function on the poset of layered permutations, that is, permutations that can be written as the direct sum of a number of decreasing permutations. More general results are presented in [BJJS11] where a formula is given for the Möbius function of all separable permutations, that is, permutations avoiding both 3142 and 2413, along with many results for decomposable permutations, that is, permutations that can be written non-trivially as direct sums. It is also shown that the absolute value of the Möbius function has an upper bound in some of these cases.

In this paper we present some of the first results for the Möbius function on a substantial class of indecomposable permutations, the only other such result seems to be in [ST10], which gives certain cases in which the Möbius function is zero. As a result of this we show that $\mu(1, \pi)$ is unbounded, which does not seem to have been established before. Our main result is a formula for the Möbius function on the interval $[1, \pi]$ for any permutation $\pi$ with at most one descent and that on such intervals the Möbius function is alternating. Note that a permutation of length $n$ with one descent is indecomposable unless it starts with 1 or ends with $n$, so our result applies to a substantial class of indecomposable permutations.

Define the subposet $\mathcal{P}_{k} \subseteq \mathcal{P}$ to be the poset containing permutations with exactly $k$ descents. In this paper we mainly treat permutations from the subposets $\mathcal{P}_{0}$ and $\mathcal{P}_{1}$. We also use the notation $\mathcal{P}_{k}^{n}$ for the set of permutations of length $n$ with exactly $k$ descents and $\mathcal{P}^{n}$ for the set of all permutations of length $n$. As we often treat the Möbius function of the intervals $[1, \pi]$ we consider the Möbius function as a function of a single variable in the form of $\mu(\pi):=\mu(1, \pi)$. An adjacency in a permutation is two letters that are consecutive in position and have consecutive increasing values. For example, 24578136 has adjacencies 45 and 78, at positions 2 and 4 . A permutation can also have a triple adjacency, or an adjacency of even greater length, as in the permutation 12456837 where there is the triple adjacency 456. The number of and positions of the adjacencies in a permutation will be key to our results. An important type of permutations from $\mathcal{P}_{1}$ are the permutations without adjacencies, which are the permutations where odd and even letters are separated from each other. We denote the even length permutations without adjacencies as $M_{n}=246 \ldots(2 n) 135 \ldots(2 n-1)$ and $W_{n}=135 \ldots(2 n-1) 246 \ldots(2 n)$ for $n>1$.

In Section 2 we prove that $\mu(\pi)=0$ for any permutation $\pi$ containing a triple adjacency. In Section 3 we prove a result relating to permutations with no adjacencies that is useful in the proof in Section 4. In Section 4 we completely classify the Möbius function on the intervals $[1, \pi]$ where $\pi$ has at most one descent. This proves the conjecture made 
in [Ste13] that $\mu(\pi)=\left(\begin{array}{c}n+1 \\ 2\end{array}\right)$ when $\pi$ is of the form $246 \ldots(2 n) 135 \ldots(2 n-1)$, which is the permutation without adjacencies $M_{n}$. This shows that $\mu(\pi)$ is unbounded in general, answering a question asked in [BJJS11], where it was shown that $|\mu(\pi)| \leqslant 1$ for all separable permutations $\pi$. In Section 5 we present additional conjectures we have not been able to prove on the Möbius function of permutations with at most one descent.

\section{The Möbius function on permutations with a triple adjacency}

In this section we present and prove a lemma stating that a permutation with a triple adjacency has a Möbius value of zero. While interesting in its own right, it is useful in proving the result in Section 4. But first we consider some notation and important points about adjacencies.

We defined an adjacency in the introduction as two letters that are consecutive in position and have consecutive increasing values. There is an analogous decreasing adjacency but we consider adjacencies to be increasing unless otherwise stated, because in $\mathcal{P}_{1}$ decreasing adjacencies are rare and do not play a role in our considerations. We denote the value of an adjacency by the value of its initial letter, so in the permutation 24578136 the adjacencies 45 and 78 have values 4 and 7 . Notice that a triple adjacency consists of two adjacencies of two letters, for example we can split 456 into 45 and 56 . When counting the adjacencies in a permutation we count adjacencies of two letters, therefore 12456837 has three adjacencies 12, 45 and 56.

Lemma 1. If a permutation $\pi$ contains a triple adjacency then $\mu(\pi)=0$.

Proof. We can easily check that $\mu(123)=0$. Now assume that the claim holds for any permutation of length $m<n$ where $m \geqslant 3$. Given a permutation $\pi \in \mathcal{P}^{n}$ with a triple adjacency, removing any of the letters of the triple adjacency from $\pi$ results in the same permutation, call this $\sigma$. Any permutation obtained from $\pi$ by removing any of the letters not in the triple adjacency still has a triple adjacency hence by the inductive hypothesis has a zero Möbius value. Hence all nonzero permutations in $[1, \pi)$ occur in $[1, \sigma]$, implying:

$$
\mu(\pi)=-\sum_{1 \leqslant z<\pi} \mu(1, z)=-\sum_{1 \leqslant z \leqslant \sigma} \mu(1, z)=0 .
$$

The result in Lemma 1 also holds for the case of decreasing triple adjacencies, with an analogous proof. We can slightly generalise this result to give the following corollary, whose proof is analogous to the proof of Lemma 1 after suitably modifying the base case:

Corollary 2. If a permutation $\pi$ contains an adjacency (increasing or decreasing) of length $k \geqslant 3$, then $\mu(12 \ldots(k-2), \pi)=0$. 


\section{The permutations with one descent and no adja- cencies}

We present a result on permutations with no adjacencies that is useful in proving the results in Section 4. Before stating the lemma, we introduce some notation and definitions along with a few remarks about the posets $\mathcal{P}_{0}$ and $\mathcal{P}_{1}$.

We say that two permutations are related if both or neither permutation begins with 1 . For example the permutations 246135 and 2357146 are related as neither begins with 1 but 246135 and 135246 are not related.

Let the increasing permutation $12 \ldots k$ be denoted $\mathbf{k}$. Notice that the poset $\mathcal{P}_{0}$ forms a chain, as for any $k \geqslant 1$ the only length $k$ permutation without a descent is the increasing permutation $\mathbf{k}$. As $\mathcal{P}_{0}$ is a chain it is easy to see that $\mu(\mathbf{k})=0$ for any $k>2$.

It is also important to note that a permutation with $k$ descents cannot contain, as a pattern, a permutation with more than $k$ descents. Therefore, in any interval $[\mathbf{1}, \pi]$, with $\pi \in \mathcal{P}_{1}$, any permutation $\lambda \in[\mathbf{1}, \pi]$ must be in $\mathcal{P}_{0} \cup \mathcal{P}_{1}$. That is, the set $\mathcal{P}_{0} \cup \mathcal{P}_{1}$ is an order ideal in $\mathcal{P}$, also called a permutation class. A permutation class can be uniquely determined by its basis, that is, the set of minimal permutations it avoids. The basis for $\mathcal{P}_{0} \cup \mathcal{P}_{1}$ can be shown to be $\{321,2143,3142\}$. We remark that the poset of permutations with at most $k$ descents, for any fixed $k$, is a permutation class, but the basis for the general case $k>1$ is much more difficult to find. A formula is given in [BF13, Theorem 4.2] which can be used to calculate the size of such a basis but this formula is rather complicated.

Recall that the permutations without adjacencies are the permutations where the odd and even letters are separated from each other. The even length permutations without adjacencies are $M_{n}=246 \ldots(2 n) 135 \ldots(2 n-1)$ and $W_{n}=135 \ldots(2 n-1) 246 \ldots(2 n)$ for $n>1$.

Lemma 3. Let $\pi \in P_{1}^{n}$ be a permutation with no adjacencies. Then $\pi$ contains, as patterns, precisely all permutations in $\mathcal{P}_{1}$ of length less than $n$ with at most two adjacencies except the following:

1. The permutations of length $n-1$ with two adjacencies.

2. The permutations of length $n-1$ with one adjacency that are not related to $\pi$.

3. The permutations of length $n-2$ with two adjacencies that are not related to $\pi$.

Proof. Let $R$ and $N$ be the subposets of $\mathcal{P}_{1}$ which contain the permutations, of length $m<n$, that are related and not related to $\pi$, respectively. Also denote the subposets of $R$ and $N$ with exactly $k$ adjacencies as $R^{k}$ and $N^{k}$, respectively. We need to prove that $\pi$ contains all permutations $\sigma \in R^{0} \cup N^{0} \cup R^{1}$, all permutations $\sigma \in R^{2} \cup N^{1}$ of length $m<n-1$ and all permutations $\sigma \in N^{2}$ of length $m<n-2$.

First consider the permutations in $R$. Note that $R^{0}$ is a chain and any permutation in $R^{0}$, of length $m<n$, can be obtained by removing the $n-m$ largest letters of $\pi$. To obtain a permutation $\sigma \in R^{1}$, of length $m<n$, where the adjacency has value $i$, 
it is necessary and sufficient to remove the letter $i+1$ from $\pi$ and then to adjust to the correct length permutation by removing the $n-m-1$ largest letters. So to create any permutation in $R^{0} \cup R^{1}$ there is only one letter that must be removed and thus all permutations in $R^{0} \cup R^{1}$ of length $m \leqslant n-1$ can be obtained from $\pi$. Now consider a permutation $\tau \in R^{2}$, of length $m<n$, with adjacencies of value $i$ and $k$. To create such a permutation, from $\pi$, we remove the letters of value $i+1$ and $k+1$ and then we adjust the length by removing the $n-m-2$ largest letters. So the permutations in $R^{2}$ require at least two letters to be removed and therefore all the permutations in $R^{2}$ of length $m \leqslant n-2$ can be obtained, but none of length $n-1$.

Now consider the permutations in $N$. Removing the letter 1 from $\pi$ creates a unique length $n-1$ permutation $\lambda$ which is in $N^{0}$. We can then apply the same argument as in the previous paragraph to $\lambda$ instead of $\pi$. Hence we can obtain all permutations in $N^{0} \cup N^{1}$ of length $m \leqslant n-2$ and all permutations in $N^{2}$ of length $m \leqslant n-3$. As $\lambda$ is the only permutation of length $n-1$ in $N^{0}$ we can get all permutations in $N^{0}$ of length $m \leqslant n-1$.

Therefore we can obtain all permutations with at most two adjacencies in $R \cup N$ except for the following: The permutations in $R^{2}$ of length $n-1$, the permutations in $N^{2}$ of lengths $n-1$ and $n-2$ and the permutations in $N^{1}$ of length $n-1$.

We provide an example of Lemma 3:

Example 4. Consider the permutation $\pi=135246$. By Lemma 3 we know the only permutations with at most two adjacencies not contained in $\pi$ are:

1. The permutations of length 5 with two adjacencies that is: $12354,41235,12534$, 34125, 12453, 31245,15234, 23415, 14523, 23145, 13452 and 21345.

2. The permutations of length 5 with one adjacency that are not related to $\pi$ that is: $35124,23514,25134$ and 24513.

3. The permutations of length 4 with two adjacencies that are not related to $\pi$ that is: 4123, 3412 and 2341.

\section{The Möbius function for permutations with one descent}

In this section we present a formula for the Möbius function on the interval $[\mathbf{1}, \pi]$ where $\pi$ is any permutation with at most one descent.

Theorem 5. Given a permutation $\pi \in \mathcal{P}_{0} \cup \mathcal{P}_{1}$, of length $n>2$, the value of $\mu(\pi)$ can be computed from the number and positions of adjacencies in $\pi$, as follows:

1. If $\pi$ begins with 12 or ends in $(n-1) n$ then $\mu(\pi)=0$.

2. If $\pi$ has a triple adjacency then $\mu(\pi)=0$.

3. If $\pi$ has more than two adjacencies then $\mu(\pi)=0$. 
4. If $\pi$ has exactly two adjacencies then:

(a) If the first adjacency has greater value than the second then $\mu(\pi)= \pm 1$,

(b) If the first adjacency has lower value than the second then $\mu(\pi)=0$.

5. If $\pi$ has exactly one adjacency, at position $i \in\{1, \ldots, n-1\}$, and the descent is at position d, then: (see item 7 for calculating the sign)

(a) If $i<d$ and $\pi_{1} \neq 1$ then $\mu(\pi)= \pm i$,

(b) If $i<d$ and $\pi_{1}=1$ then $\mu(\pi)= \pm(i-1)$,

(c) If $i>d$ and $\pi_{n} \neq n$ then $\mu(\pi)= \pm(n-i)$,

(d) If $i>d$ and $\pi_{n}=n$ then $\mu(\pi)= \pm(n-i-1)$.

6. If $\pi$ has no adjacencies then:

(a) If $n$ is even and $\pi_{1}=1$, that is $\pi=W_{\frac{n}{2}}$, then $\mu(\pi)=-\left(\begin{array}{c}\frac{n}{2} \\ 2\end{array}\right)$,

(b) If $n$ is even and $\pi_{1}=2$, that is $\pi=M_{\frac{n}{2}}$, then $\mu(\pi)=-\left(\begin{array}{c}\frac{n}{2}+1 \\ 2\end{array}\right)$,

(c) If $n$ is odd then $\mu(\pi)=\left(\begin{array}{c}\frac{n+1}{2} \\ 2\end{array}\right)$.

7. If $\mu(\pi) \neq 0$ then $\mu(\pi)$ is positive if and only if $n$ is odd.

Before proving Theorem 5 we make some remarks:

- Each permutation with one descent falls into at least one of the above classes.

- The above result agrees on permutations covered by more than one class. These cases are:

- A permutation with one adjacency and beginning with 12 or ending with $(n-$ 1) $n$ has zero Möbius value by both part 1 and part 5 .

- A permutation with 12 at the beginning and $(n-1) n$ at the end has zero Möbius value by both part 1 and part $4 \mathrm{~b}$.

- A triple adjacency can be treated as two consecutive adjacencies and the result states the Möbius value is zero by part 2 and part $4 \mathrm{~b}$.

- It is possible for a permutation to fall into all three of the first cases, such as 12354, and such a permutation has zero Möbius value according to all three cases. 
For part 1 of Theorem 5 , a permutation that begins with 12 or ends with $(n-1) n$ is decomposable so the proof follows directly from Corollary 3 in [BJJS11] and part 2 follows from Lemma 1.

We prove the remaining parts of Theorem 5 using an inductive argument throughout the following subsections. For a base case we need to consider all permutations of length $3 \leqslant n \leqslant 6$. We know certain permutations have zero Möbius value by the already proven parts 1 and 2 of Theorem 5 So we can leave such permutations. We now list the remaining permutations of length $3 \leqslant n \leqslant 6$ with one descent along with their calculated Möbius value and which case of Theorem 5 they fall into: $\mu(34125)=\mu(14523)=1(4 a)$, $\mu(3412)=\mu(145236)=\mu(256134)=\mu(346125)=\mu(356124)=-1(4 a), \mu(235614)=$ $\mu(236145)=\mu(361245)=0(4 b), \mu(231)=\mu(312)=\mu(13425)=\mu(14235)=\mu(23514)=$ $\mu(25134)=1(5), \mu(1423)=\mu(3124)=\mu(1342)=\mu(2314)=\mu(134625)=\mu(136245)=$ $\mu(235146)=\mu(251346)=-1(5), \mu(24513)=\mu(35124)=2(5), \mu(245136)=\mu(351246)=$ $\mu(146235)=\mu(135624)=-2(5), \mu(132)=\mu(213)=1(6), \mu(1324)=\mu(2413)=-1(6)$, $\mu(13524)=\mu(24135)=3(6), \mu(135246)=-3(6 a), \mu(246135)=-6(6 b)$.

It is straightforward to check that these results agree with Theorem 5 .

The reason it is necessary to check the base case up to length $n=6$ is so we can use Lemma 8 to cancel out the Möbius values of sets of permutations in the intervals we consider.

From now on we assume that any permutation in $\mathcal{P}_{0} \cup \mathcal{P}_{1}$, of length less than $n$, where $n>6$, satisfies the claims in Theorem 5 and we prove that Theorem 5 then holds for permutations with at most one descent of length $n$ and thus for any length. When referencing the induction hypothesis we add the part of Theorem 5 being referenced in brackets, for example (5.6a) for Theorem 5 part 6 a.

By our inductive hypothesis (5.3) we can see that any nonzero permutation of length $m<n$ can have at most two adjacencies. If we combine this with Lemma 3 we see that a permutation of length $m<n$ with no adjacencies contains all nonzero permutations of length at most $m-3$.

\subsection{The structure of the proof}

The remaining parts of the proof of Theorem 5 all follow a similar schema, which is outlined as follows:

1. Consider $\pi \in \mathcal{P}_{1}^{n}$.

2. Remove one letter from each adjacency in $\pi$ or the largest letter if $\pi$ has no adjacencies. This leaves a permutation $\lambda$ with no adjacencies.

3. By the definition of the Möbius function, $\sum_{\sigma \in[\mathbf{1}, \lambda]} \mu(\sigma)=0$.

4. Now we can compute $\mu(\pi)$ using $\mu(\pi)=-\sum_{\substack{\sigma<\pi \\ \sigma \nless \lambda}} \mu(\sigma)$. 
We develop this schema in detail for the proof of Proposition 1 in the following subsection and then, as they are quite similar, the remaining parts of the proof are done in less detail.

We present two lemmas which we frequently reference throughout the proof:

Lemma 6. Let $\sigma \in \mathcal{P}_{1}^{m}$, where $m<n$, be a nonzero permutation satisfying either one of the following conditions:

1. Has exactly one adjacency, which is neither 12 nor $(m-1) m$.

2. Has exactly two adjacencies at least one of which is neither 12 nor $(m-1) m$.

Then $\sigma$ contains a length $m-1$ permutation $\lambda$ with the same number of adjacencies as $\sigma$ such that $\mu(\lambda)+\mu(\sigma)=0$.

Proof. If $\sigma$ has exactly one adjacency, at location $i$, then either this adjacency is before or after the descent. If the adjacency is before the descent, then by the induction hypothesis (5.5) the Möbius value of $\sigma$ is a function of $i$. We know $m$ must be to the right of $i$ so removing $m$ creates a length $m-1$ permutation $\lambda$ with exactly one adjacency at location $i$, so by the induction hypothesis (5.5 and 5.7) $\mu(\sigma)=-\mu(\lambda)$. If the adjacency is after the descent then removing the letter 1 gives an analogous argument. This completes the first case.

If $\sigma$ has exactly two adjacencies we can remove either the letter 1 or $m$ which gives a length $m-1$ permutation $\lambda$ which has two adjacencies of the same relative sizes as the adjacencies in $\sigma$. By the induction hypothesis (5.7) the sign of the Möbius function is alternating, therefore $\mu(\lambda)=-\mu(\sigma)$.

Example 7. 1. Consider the permutation 13425 which is of the first form in Lemma 6 . Removing the letter 5 gives the permutation 1342. We compute the Möbius values of these permutations as $\mu(13425)=1$ and $\mu(1342)=-1$.

2. Consider the permutation 24781356 which is of the second form in Lemma 6 . Removing the letter 1 gives the permutation 1367245. We compute the Möbius values of these permutations as $\mu(24781356)=-1$ and $\mu(1367245)=1$.

We can use Lemma 6 to show that the Möbius values of certain sets of permutations sum to zero.

Lemma 8. Take a set $\Delta^{1}$ of $k$ nonzero permutations from $\mathcal{P}_{1}^{m}$, where $4<m<n$, all with $t>0$ adjacencies and where none of the adjacencies is 12 or $(m-1) m$. Then we can construct the following sets:

- A set $\Delta^{2}$ of $2 k$ permutations from $\mathcal{P}_{1}^{m-1}$ with exactly $t$ adjacencies.

- A set $\Delta^{3}$ of $k$ permutations from $\mathcal{P}_{1}^{m-2}$ with exactly $t$ adjacencies.

Also the sum of the Möbius values of all the permutations in $\Delta^{1} \cup \Delta^{2} \cup \Delta^{3}$ is zero. 
Proof. From each permutation in $\Delta^{1}$ we have two options: We can remove the letter 1 or the letter $m$. Assume first that the removal of either of these letters from any of the permutations does not remove the descent from the permutation, then it is easy to see that this does not create or remove an adjacency. So to create $\Delta^{2}$ we get two permutations for each permutation in $\Delta^{1}$ by removing either 1 or $m$. To create $\Delta^{3}$ we remove, from each permutation in $\Delta^{1}$, both 1 and $m$. It is easy to see that, as we are only removing the letters 1 and $m$, the permutations in the union of $\Delta^{2}$ and $\Delta^{3}$ are distinct. This concludes the proof of the first part of the lemma.

To show that the Möbius values sum to zero we can apply Lemma 6. Recall that a permutation with $t>2$ adjacencies has Möbius value zero by the induction hypothesis (5.3). As $\Delta^{1}$ only contains nonzero permutations any permutation $\lambda \in \Delta^{1}$ must be of one of the forms in Lemma 6. First suppose it is of the first form, that is, it has one adjacency, and suppose this adjacency is before the descent. We can pair $\lambda$ with the permutation obtained by removing the letter $m$ from $\lambda$ and their Möbius values sum to zero. Then, given the permutation $\lambda^{1} \in \Delta^{2}$ obtained by removing the letter 1 from $\lambda$, we can pair this with the permutation $\lambda^{1, m} \in \Delta^{3}$ obtained by removing 1 and $m$ from $\lambda$. By Lemma 6 we know the Möbius values of these two permutations sum to zero. We can do this for each permutation in $\Delta^{1}$, which completes this case. An analogous argument applies to the case where $\lambda$ has an adjacency after the descent or has two adjacencies.

If the removal of the letter 1 or $m$ results in the removal of the descent from one of the permutations then we apply an analogous argument to entire proof above. In this argument we must account for the fact that for each permutation of this form there are two permutations that are increasing permutations of length greater than 2. As such, these permutations contain a triple adjacency and will have zero Möbius value and the Möbius value of the remaining permutations cancel as above.

\subsection{Theorem 5 part 3}

Recall that we are assuming Theorem 5 is true for any permutations of length $m<n$. We now consider part 3 of Theorem 5 for permutations of length $n$.

Proposition 1. A permutation $\pi \in \mathcal{P}_{1}^{n}$ with more than two adjacencies has $\mu(\pi)=0$.

Proof. Suppose $\pi$ has $k>2$ adjacencies also suppose none of the adjacencies are 12 or $(n-1) n$ and there are no triple adjacencies. Then, by the inductive hypothesis (5.3), $\pi$ contains no nonzero permutations of length greater than $n-k+2$. There is a unique length $n-k$ permutation $\lambda$ contained in $\pi$ with no adjacencies. Let us ignore $\lambda$ along with any other permutation in $[\mathbf{1}, \lambda]$, since their contributions to the Möbius value of $\pi$ sum to zero. Then we can use Lemma 3 to consider the remaining permutations, that are possibly nonzero, occurring in $\pi$ :

- Of length $n-k+2$ there remain $s=\left(\begin{array}{l}k \\ 2\end{array}\right)$ permutations with two adjacencies, call these $\Gamma^{0}=\left\{\gamma_{1}^{0}, \ldots, \gamma_{s}^{0}\right\}$, where each of the $\gamma_{i}^{0}$ 's is obtained by removing a letter from all but two of the adjacencies in $\pi$. 
- Of length $n-k+1$ there remain:

- $k$ nonzero permutations with one adjacency, call these $\Delta^{0}=\left\{\delta_{1}^{0}, \ldots, \delta_{k}^{0}\right\}$.

$-2\left(\begin{array}{l}k \\ 2\end{array}\right)$ permutations with two adjacencies obtained by removing the letter 1 or the largest letter from each of the $\gamma_{i}^{0}$ 's, call these $\Gamma^{1}=\left\{\gamma_{1}^{1}, \ldots, \gamma_{2 s}^{1}\right\}$.

- Of length $n-k$ there remain:

- All the permutations related to $\pi$ that have two adjacencies, where at least one of the adjacencies is an original adjacency in $\pi$, call this set of permutations $\Omega^{0}$.

- $\left(\begin{array}{l}k \\ 2\end{array}\right)$ permutations not related to $\pi$ that have two adjacencies, both occurring in $\pi$, call these $\Gamma^{2}=\left\{\gamma_{1}^{2}, \ldots, \gamma_{s}^{2}\right\}$.

- $2 k$ permutations with one adjacency obtained by removing the letter 1 or the largest letter from the $\delta_{i}^{0}$ 's, call these $\Delta^{1}=\left\{\delta_{1}^{1}, \ldots, \delta_{2 k}^{1}\right\}$.

- Of length $n-k-1$ there remain:

- All permutations with two adjacencies, where at least one of the adjacencies is an original adjacency in $\pi$, call this set of permutations $\Omega^{1}$.

$-k$ permutations with one adjacency obtained by removing the letter 1 and the largest letter from each $\delta_{i}^{0}$, call these $\Delta^{2}=\left\{\delta_{1}^{2}, \ldots, \delta_{k}^{2}\right\}$.

- Of length $n-k-2$ all permutations not related to $\pi$ with two adjacencies, where at least one of the adjacencies is an original adjacency in $\pi$, call this set of permutations $\Omega^{2}$.

Note that by Lemma 8 the Möbius values in $\Delta^{0} \cup \Delta^{1} \cup \Delta^{2}$ sum to zero and the same is true of $\Gamma^{0} \cup \Gamma^{1} \cup \Gamma^{2}$ and $\Omega^{0} \cup \Omega^{1} \cup \Omega^{2}$. We know these sets satisfy the length condtions in Lemma 8 because the maximum number of adjacencies is $\frac{n-2}{2}$, this is because the letters 1 and $n$ are not in adjacencies and there are no triple adjacencies, which implies $n-k \geqslant \frac{n+2}{2} \geqslant 4.5>4$. This implies $\mu(\pi)=0$ and completes this case.

Now suppose one of the adjacencies in $\pi$ is 12 or $(n-1) n$. If these adjacencies occur at the beginning or end, respectively, then this reduces to part 1 of Theorem 5 . It is also possible that one of these adjacencies occurs directly after or before the descent in which case the proof follows from the proof above with minor modifications. These modifications arise from the fact that removing the letter 1 from $\pi$ is equivalent to removing the adjacency 12 and likewise with the letter $n$ and the adjacency $(n-1) n$. In certain cases, this may result in $n-k \ngtr 4$, and we must apply Lemma 6 to get the desired cancellation.

\subsection{Theorem 5 part 4}

Proposition 2. Consider a permutation $\pi \in \mathcal{P}_{1}^{n}$ with exactly two adjacencies, at positions $k$ and $i$. If the first adjacency has greater value than the second then $\mu(\pi)= \pm 1$, otherwise $\mu(\pi)=0$. 
Proof. If $\pi$ begins with 12 or ends with $(n-1) n$, then $\mu(\pi)=0$ by part 1 of Theorem 5 . Now consider the case $\pi$ does not contain both the adjacencies 12 and $(n-1) n$.

Removing the letters $\pi_{i}$ and $\pi_{k}$ results in a permutation $\lambda$, of length $n-2$, with no adjacencies. As the Möbius values of all the permutations in $[\mathbf{1}, \lambda]$ sums to zero we can ignore any permutation in said interval. Now use Lemma 3 and consider the remaining permutations. By Lemma 8 the Möbius values of the remaining permutations with one adjacency sum to zero. Split the remaining permutations with two adjacencies into two sets $A$ and $B$, where $A$ are those obtained from $\pi$ by removing the letters 1 or $n$ (or both) and $B$ are those obtained from $\pi$ by removing a letter from an adjacency and then removing another letter to create a new adjacency that does not occur in $\pi$. As the largest permutations in $B$ are of length $n-2>4$ we can apply Lemma 8 to see that the Möbius values of the permutations in $B$ sum to zero.

This just leaves us to consider $A$. First assume $\pi$ doesn't have the adjacencies 12 or $(n-1) n$ directly after or before the descent. Then $A$ contains the following permutations with two adjacencies:

- A permutation $\delta$ of length $n-1$, obtained by removing the letter 1 from $\pi$.

- A permutation $\tau$ of length $n-1$, obtained by removing the letter $n$ from $\pi$.

- A permutation $\sigma$ of length $n-2$, obtained by removing letters 1 and $n$ from $\pi$.

By the inductive hypothesis (5.4 and 5.7) it is clear that $\mu(\tau)+\mu(\sigma)=0$. This means that $\mu(\pi)=-\mu(\delta)$. The relative values of the adjacencies in $\pi$ are the same as in $\delta$ so, if the first adjacency has greater value than the second then $\mu(\pi)=-\mu(\delta)= \pm 1$, otherwise $\mu(\pi)=-\mu(\delta)=0$. This completes the first case.

Now consider the case when $\pi$ contains the adjacency 12 but not $(n-1) n$, then $A$ only contains $\tau$ and $\mu(\pi)=-\mu(\tau)$. Similarly when $\pi$ contains the adjacency $(n-1) n$ but not 12 , then $A$ only contains $\delta$ and $\mu(\pi)=-\mu(\delta)$. The result then follows by evaluating the value of $\delta$ or $\tau$. This completes this case.

Finally consider the case $\pi$ contains both adjacencies 12 and $(n-1) n$ and with $(n-1) n$ occurring before 12 , in this case there are no permutations in the set denoted $A$ above and not all the permutations with one adjacency cancel. So we repeat the argument above considering the permutations with one adjacency.

Remark: Note that the Möbius value in the above proof is computed as a negation of a permutation of length one less. Hence the Möbius value is alternating in the case of permutations with two adjacencies.

\subsection{Theorem 5 part 5}

Proposition 3. Consider a permutation $\pi \in \mathcal{P}_{1}^{n}$ which has exactly one adjacency at position $i$ and the descent at position $d$. Then:

1. If $i<d$ and $\pi_{1} \neq 1$ then $\mu(\pi)= \pm i$, 
2. If $i<d$ and $\pi_{1}=1$ then $\mu(\pi)= \pm(i-1)$,

3. If $i>d$ and $\pi_{n} \neq n$ then $\mu(\pi)= \pm(n-i)$,

4. If $i>d$ and $\pi_{n}=n$ then $\mu(\pi)= \pm(n-i-1)$.

Proof. If $\pi$ begins with 12 or ends with $(n-1) n$, then $\mu(\pi)=0$ by part 1 of Theorem 5 . Next, we consider the case where $\pi$ doesn't have the adjacencies 12 or $(n-1) n$ directly before or after the descent.

Removing $\pi_{i}$ from $\pi$ creates a permutation $\lambda$ with no adjacencies and we can ignore the interval $[\mathbf{1}, \lambda]$ as the Möbius values sum to zero by definition. We can apply Lemma 8 to the remaining permutations with two adjacencies to see that their Möbius values sum to zero. By Lemma 3 this leaves us to consider three permutations with one adjacency:

- Of length $n-1$ there remain two permutations with one adjacency, obtained by removing the letters 1 or $n$, call these $\sigma_{1}$ and $\sigma_{2}$ respectively.

- Of length $n-2$ there remains one permutation with one adjacency not related to $\pi$. This is obtained by removing the letters 1 and $n$ from $\pi$, call this $\delta$.

We consider the four cases in the statement of the proposition and obtain the Möbius value from the induction hypothesis (5.5):

- If $i<d$ then $\mu\left(\sigma_{1}\right)+\mu(\delta)=0$. Hence $\mu(\pi)=-\mu\left(\sigma_{2}\right)$ which gives:

1. If $\pi_{1} \neq 1$ then $\mu(\pi)=-\mu\left(\sigma_{2}\right)= \pm i$.

2. If $\pi_{1}=1$ then $\mu(\pi)=-\mu\left(\sigma_{2}\right)= \pm(i-1)$.

- If $i>d$ then $\mu\left(\sigma_{2}\right)+\mu(\delta)=0$. Hence $\mu(\pi)=-\mu\left(\sigma_{1}\right)$ which gives:

3. If $\pi_{n} \neq n$ then $\mu(\pi)=-\mu\left(\sigma_{1}\right)= \pm(n-i)$.

4. If $\pi_{n}=n$ then $\mu(\pi)=-\mu\left(\sigma_{1}\right)= \pm(n-i-1)$.

This completes this case of the proof.

If $\pi$ contains the adjacency 12 or $(n-1) n$ then removing the letter $n$ or 1 , respectively, gives a permutation with one adjacency $\alpha$. The Möbius values of all the other permutations sum to zero by Lemma 8, so $\mu(\pi)=-\mu(\alpha)$. Evaluating the four different cases of the proposition and using the inductive hypothesis (5.5) to get $\mu(\alpha)$ completes the proof.

Remark: Note that in the above proof for each case the Möbius value of $\pi$ is a negation of a permutation of length one less. Therefore the sign of the Möbius value is alternating for all permutations with exactly one adjacency. 


\subsection{Theorem 5 part 6}

Proposition 4. Let $\pi$ be a permutation in $\mathcal{P}_{1}^{n}$ with no adjacencies. Then:

1. If $n$ is even and $\pi_{1}=1$, that is $\pi=W_{\frac{n}{2}}$, then $\mu(\pi)=-\left(\begin{array}{c}\frac{n}{2} \\ 2\end{array}\right)$,

2. If $n$ is even and $\pi_{1}=2$, that is $\pi=M_{\frac{n}{2}}$, then $\mu(\pi)=-\left(\begin{array}{c}\frac{n}{2}+1 \\ 2\end{array}\right)$,

3. If $n$ is odd then $\mu(\pi)=\left(\begin{array}{c}\frac{n+1}{2} \\ 2\end{array}\right)$.

Proof. First note that $\pi$ contains a permutation $\lambda$, with no adjacencies, of length $n-1$, obtained by removing the largest letter from $\pi$. As the Möbius values of all the permutations in $[\mathbf{1}, \lambda]$ sum to zero we can ignore any permutation in said interval. By Lemma 3 this leaves us to consider the following permutations which occur in $\pi$ :

- Of length $n-1$ there remain:

- One permutation with no adjacencies obtained by removing the letter 1 from $\pi$.

- The permutations with one adjacency each obtained by removing a letter from $\pi$, excluding the letters 1 and $n$.

- Of length $n-2$ there remain:

- The permutations not related to $\pi$ with one adjacency. These are obtained by removing the letter 1 from each of the permutations with one adjacency of length $n-1$ listed above.

- All permutations of length $n-2$ related to $\pi$ with two adjacencies.

- Of length $n-3$ there remain the permutations not related to $\pi$ with two adjacencies.

First consider the case $n$ is even and $\pi_{1}=1$, that is when $\pi=13 \ldots(n-1) 24 \ldots n=$ $W_{\frac{n}{2}}$. We will consider the permutations in $\left[1, W_{\frac{n}{2}}\right]$ based on number of adjacencies, and when needed, by the number removed to create an adjacency. We start with the nonzero permutations with two adjacencies. Note that all the length $n-3$ permutations with two adjacencies are obtained from the length $n-2$ permutations with two adjacencies by removing the letter 1 . We can then apply Lemma 6 to see that the Möbius values of the permutations with two adjacencies sum to zero. We can repeat this argument with the permutations with one adjacency obtained from $\pi$ by removing any of the letters $3,4, \ldots,(n-1)$ to see that these cancel with the permutations of length $n-2$ with one adjacency. This leaves the permutations obtained from $\pi$ by removing the letters 2 and 1 , respectively. The first is of the form $124 \ldots(n-2) 3 \ldots(n-1)$ and begins with 12 so has zero Möbius value by part 1 of Theorem 5 . The second is $24 \ldots(n-2) 13 \ldots(n-1)$ which by the induction hypothesis (5.6c) has Möbius value $\left(\frac{\frac{n-1+1}{2}}{2}\right)$, which implies $\mu\left(W_{\frac{n}{2}}\right)=-\left(\begin{array}{c}\frac{n}{2} \\ 2\end{array}\right)$. 
In the case $n$ is odd and $\pi_{1}=1$, the argument is analogous. We find the permutation $24 \ldots n 13 \ldots(n-1)$ has Möbius value $-\left(\frac{n-1}{2}+1\right)$, which implies $\mu(\pi)=\left(\begin{array}{c}n+1 \\ 2\end{array}\right)$.

Next consider the case where $n$ is even and $\pi_{1}=2$, that is $\pi=24 \ldots n 13 \ldots(n-1)=$ $M_{\frac{n}{2}}$. First we consider permutations of length $n-1$ with one adjacency formed by removing one of the letters $3,4, \ldots,(n-1)$. We can apply Lemma 6 to see that the Möbius value of all but one of these cancel with all but one of the length $n-2$ permutations with one adjacency. The only remaining length $n-2$ permutation is $124 \ldots(n-2) 35 \ldots(n-3)$ which has zero Möbius value by part 1 of Theorem 5 . The only remaining length $n-1$ permutation is $24 \ldots(n-2)(n-1) 13 \ldots(n-3)$ which by the induction hypothesis $(5.5 \mathrm{a})$ has Möbius value $\frac{n}{2}-1$.

Now consider the remaining permutations with two adjacencies. The permutation with the triple adjacency 123 contributes zero to the Möbius value by part 1 of Theorem 5. Removing the letter 2 and any letter $i>3$ from $\pi$ results in a permutation with adjacency 12 immediately after the descent. If $i$ is even then the larger adjacency also appears after the descent so such a permutation contributes zero to the Möbius value by the induction hypothesis (5.4b). If $i$ is odd then the adjacency appears before the descent. Since each such permutation has Möbius value -1 by the induction hypothesis (5.4a), and $i$ is an odd number between 5 and $n$, the sum of the Möbius values of these permutations is $-\frac{n}{2}+2$. We can apply Lemma 6 to cancel all of the other permutations with two adjacencies in a similar way to the case $\pi_{1}=1$ above.

We must also consider the Möbius values of the permutations found by removing 2 or 1 from $\pi$. The permutation $35 \ldots(n-1) 124 \ldots(n-2)$ has Möbius value $\frac{n}{2}-1$ by the induction hypothesis (5.5c). The permutation $13 \ldots(n-1) 24 \ldots(n-2)$ has Möbius value $\left(\begin{array}{c}\frac{n}{2} \\ 2\end{array}\right)$ by the induction hypothesis (5.6c). The Möbius value of $\pi$ is given by the negation of the sum of the Möbius values of the permutations it contains, so we sum the above values and negate which gives:

$$
\mu(\pi)=-\left(\left(\begin{array}{c}
\frac{n}{2} \\
2
\end{array}\right)+2\left(\frac{n}{2}-1\right)-\frac{n}{2}+2\right)=-\left(\begin{array}{c}
\frac{n}{2}+1 \\
2
\end{array}\right) .
$$

Finally we consider the case where $n$ is odd and $\pi_{1}=2$, that is, $\pi=24 \ldots(n-$ $1) 13 \ldots n$. The argument proceeds in an analogous manner to the previous case, except the sum of the Möbius values of the permutations with two adjacencies is $\frac{n-1}{2}-2$ and the Möbius values of the three permutations with one adjacency are $-\frac{n}{2}+1,-\frac{n}{2}+1$ and $\left(\frac{n-1}{2}\right)$, resulting in $\mu(\pi)=\left(\frac{n+1}{2}\right)$.

\section{Remark:}

- The nice form of the result in Proposition 4 raises the question of a direct combinatorial proof. We expect to present such a proof in the forthcoming paper [Smi14] which analyses topological properties of some intervals in the poset $\mathcal{P}$.

- Notice that in the above cases the Möbius value is positive if and only if $n$ is odd. Therefore the Möbius value is alternating. 


\subsection{Finishing the proof of Theorem 5}

Notice that the remarks after Propositions 2, 3 and 4 show that the Möbius value is alternating for all nonzero permutations, which implies the Möbius value is positive if and only if $n$ is odd. This proves part 7 of Theorem 5. We have shown that if the classification of Theorem 5 holds for all permutations, of length less than $n$, with at most one descent, then it also holds for $n$. By induction, that completes the proof of Theorem 5 . Parts 5 and 6 of Theorem 5 give us the following important corollary:

Corollary 9. On the poset $\mathcal{P}$ the function $\mu(\pi)$ is unbounded.

\section{Conjectures on the Möbius function for intervals of permutations with at most one descent}

So far we have mainly concentrated on intervals of the form $[\mathbf{1}, \pi]$. We now consider permutations where we allow the permutation 1 to change. We see that this change increases the complexity of computing the Möbius function quite drastically especially in the second conjecture we present, but also leads to some interesting results relating to the Möbius function being dependant on whether a permutation is separable.

\subsection{The Möbius function on the intervals $\left[\sigma, M_{n}\right]$ and $\left[\sigma, W_{n}\right]$}

In this subsection we examine intervals $[\sigma, \pi]$ where $\pi$ is one of the two permutations of even length with no adjacencies and $\sigma \in \mathcal{P}_{1}$. Recall that these permutations with no adjacencies are denoted $M_{n}=24 \ldots(2 n) 13 \ldots(2 n-1)$ and $W_{n}=13 \ldots(2 n-1) 24 \ldots(2 n)$. This leads us to the following conjecture which has been checked by computer to hold for any pair $(m, n)$ where $m<12$ and $n<7$ :

Conjecture 10. Given a permutation $\sigma \in \mathcal{P}_{1}^{m}$, let $i$ be the number of adjacencies in $\sigma$. If $\sigma \leqslant \pi$ where $\pi \in\left\{M_{n}, W_{n}\right\}$ we have the following:

- If $\sigma$ is separable, then:

$$
\begin{aligned}
& \diamond \mu\left(\sigma, M_{n}\right)= \pm\left(\begin{array}{c}
n+1 \\
m
\end{array}\right), \\
& \diamond \mu\left(\sigma, W_{n}\right)= \pm\left(\begin{array}{c}
n+m-i-2 \\
m
\end{array}\right) .
\end{aligned}
$$

- If $\sigma$ is not separable:

$$
\mu(\sigma, \pi)= \pm\left(\begin{array}{c}
n+\left\lfloor\frac{m-i-a}{2}\right\rfloor \\
m
\end{array}\right)
$$

where $a=\left\{\begin{array}{ll}0, & \text { if } \sigma \text { and } \pi \text { are related } \\ 1, & \text { otherwise }\end{array}\right.$. 
Also the Möbius value is positive if and only if $m$ is even.

Recall that when considering an adjacency of length $k$ we regard it as $k-1$ individual adjacencies.

Notice that Conjecture 10 only deals with intervals $[\sigma, \pi]$ where $\pi$ is of even length. In Theorem 5 we can see that changing $\pi$ between odd and even length has little effect on the Möbius function. In Conjecture 10, on the other hand, there is a substantial difference between the odd and even case.

\subsection{The Möbius function on the interval $\left[M_{m}, \pi\right]$}

We can reverse the idea in subsection 5.1 and consider intervals $[\sigma, \pi]$ where $\sigma$ is a permutation without adjacencies and $\pi \in \mathcal{P}_{0}^{n} \cup \mathcal{P}_{1}^{n}$. In this subsection we conjecture a formula for the Möbius function on such intervals. This formula is somewhat complicated, but turns out to be computationally efficient, compared to the brute force method of computing from the recursive formula for the Möbius function. Before stating the result we define a few statistics on $\pi$ :

- Let $a$ be the number of adjacencies in $\pi$.

- Set $\hat{n}=\left\{\begin{array}{ll}n-1, & \text { if } \pi_{n}=n \\ n, & \text { otherwise }\end{array}\right.$.

- Let the set $A=\left\{i_{1}, \ldots, i_{a}\right\}$ be the ordered sequence of the values of the adjacencies in $\pi$. Also add to $A$ two phantom adjacencies $i_{0}$ and $i_{a+1}$ which occur before and after the descent, respectively, with values:

$$
i_{0}=\left\{\begin{array}{ll}
-1, & \text { if } \pi_{1} \neq 1 \\
0, & \text { otherwise }
\end{array} \text { and } i_{a+1}=\hat{n}+1 .\right.
$$

- A function:

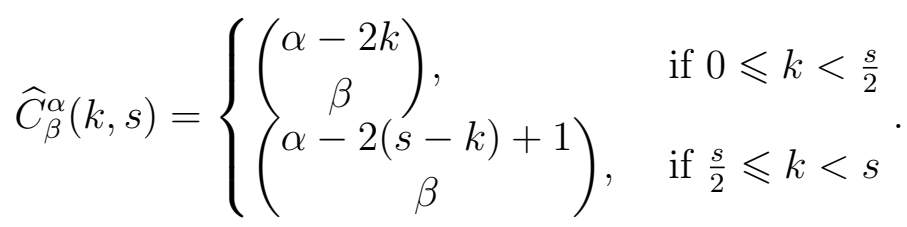

- A sequence $\hat{J}=\left\{\hat{j}_{0}, \ldots, \hat{j}_{a}\right\}$ where:

$$
\hat{j_{k}}=\left\lfloor\frac{i_{k+1}-i_{k}-2}{2}\right\rfloor .
$$

- Split $\hat{J}$ into two sequences $j^{a}$ and $j^{b}$ in the following way:

$$
\left\{\begin{array}{ll}
\hat{j_{k}} \in j^{a}, & \text { if } i_{k} \text { and } i_{k+1} \text { occur on the same side of the descent } \\
\hat{j_{k}} \in j^{b}, & \text { otherwise }
\end{array} .\right.
$$


- Set $s=\sum_{t=0}^{a} \hat{j}_{t}$.

- Trim $j^{a}$ and $j^{b}$ in the following way:

1. If $j^{a}$ is empty remove the largest element from $j^{b}$ and set $\epsilon=0$,

2. If $j^{a}$ is not empty let $\max _{j^{a}}$ be the largest element in $j^{a}$ and remove it from $j^{a}$, then set $\epsilon=\max _{j^{a}}-\sum_{t} j_{t}^{a}$,

3. Then set $\alpha=\left|j^{a}\right|$ and $\beta=\left|j^{b}\right|$,

4. Remove all zero elements from both sequences and if this results in $j^{b}$ being empty set $\epsilon=0$,

5. Finally sort $j^{a}$ into ascending order and $j^{b}$ into decreasing order.

- Define the function $s_{\theta}(\kappa, \tau)=\sum_{t=\kappa}^{\tau} j_{t}^{\theta}$.

- Set $\lambda=\left\lceil\frac{\hat{n}}{2}\right\rceil+m-\left\lceil\frac{5 a}{2}\right\rceil+\beta-t$ and $\sigma=2 m-2 a+\beta$

where $t= \begin{cases}1, & \text { if } \pi_{1}=1 \text { and } n \text { is even and } \pi_{n}=n \\ 1, & \text { if } \pi_{1}=1 \text { and } n \text { is odd and } \pi_{n} \neq n \\ 0, & \text { otherwise }\end{cases}$

For an example of these statistics see Example 12 below.

We can now state the conjecture which has been checked by computer tests to hold for all pairs $(m, n)$ where $m<6$ and $n<12$ :

Conjecture 11. Consider the interval $\left[M_{m}, \pi\right]$ where $\pi \in \mathcal{P}_{0}^{n} \cup \mathcal{P}_{1}^{n}$ and $\lambda, \sigma, j^{a}, j^{b}, \epsilon, s$, $s_{\theta}$ and $\widehat{C}$ are all as defined above, then:

If $\pi$ begins with 12, ends with $(n-1) n$ or contains a triple adjacency $\mu\left(M_{m}, \pi\right)=0$, otherwise:

$$
\begin{aligned}
\left|\mu\left(M_{m}, \pi\right)\right| & =\left(\begin{array}{c}
\lambda \\
\sigma
\end{array}\right)-\sum_{\tau=0}^{\left|j^{b}\right|-1} \sum_{\gamma=0}^{\tau} \sum_{\omega=\tau-\gamma}^{j_{\gamma}^{b}+s_{b}\left(\tau+1,\left|j^{b}\right|-1\right)-1} \widehat{C}_{\sigma-\tau-1}^{\lambda-\tau-2}(\omega, s) \\
& +\sum_{\tau=0}^{\left|j^{a}\right|-1}\left[\sum_{\gamma=1}^{j_{\tau}^{a}+s_{a}(0, \tau-1)} \widehat{C}_{\sigma-\left|j^{b}\right|-\tau}^{\lambda-\left|j^{b}\right|-\tau}(\gamma, s+1)+\sum_{\omega=1}^{\epsilon} \widehat{C}_{\sigma-\left|j^{b}\right|-\tau}^{\lambda-\left|j^{b}\right|-\tau}(\omega+1, s+1)\right]
\end{aligned}
$$

Also the sign of $\mu$ is positive if and only if $n$ is even.

Whilst Conjecture 11 is rather complicated it is significantly more computationally efficient than computing the Möbius function from its recursive definition. To see this consider the following example, for an interval of rank 20, whose computation from the recursive definition would take enormous time even on a fast computer: 
Example 12. Consider the interval

$$
I=[24681357,24679121416182123242628135810111315171920222527] .
$$

We compute $\mu(I)$ using Conjecture 11, first extracting the following statistics from $I$ :

- $a=4, m=4$ and $\hat{n}=28$,

- $A=\{-1,6,10,19,23,29\}$ and $\hat{J}=\{2,1,3,1,2\}$,

- Before trimming: $j^{a}=\{2,3\}$ and $j^{b}=\{1,1,2\}$,

- After trimming: $j^{a}=\{2\}, j^{b}=\{2,1,1\}, \epsilon=1, \alpha=1$ and $\beta=3$,

- $s=9, \lambda=11$ and $\sigma=3$.

Putting this into the formula of Conjecture 11 we get:

$$
\begin{aligned}
\mu(I) & =\left(\begin{array}{c}
11 \\
3
\end{array}\right)-\sum_{\tau=0}^{2} \sum_{\gamma=0}^{\tau} \sum_{\omega=\tau-\gamma}^{j_{\gamma}^{b}+s_{b}\left(\tau+1,\left|j^{b}\right|-1\right)-1} \widehat{C}_{2-\tau}^{9-\tau}(\omega, 9) \\
& +\sum_{\tau=0}^{0}\left[\sum_{\gamma=1}^{j_{\tau}^{a}+s_{a}(0, \tau-1)} \widehat{C}_{0-\tau}^{8-\tau}(\gamma, 10)+\sum_{\omega=1}^{1} \widehat{C}_{0-\tau}^{8-\tau}(\omega+1,10)\right] \\
& =\left(\begin{array}{c}
11 \\
3
\end{array}\right)-\sum_{\omega=0}^{4} \widehat{C}_{2}^{9}(\omega, 9)-\left[\sum_{\omega=1}^{2} \widehat{C}_{1}^{8}(\omega, 9)+\sum_{\omega=0}^{1} \widehat{C}_{1}^{8}(\omega, 9)\right] \\
& -\sum_{\omega=0}^{0} \widehat{C}_{0}^{7}(\omega, 9)+\sum_{\gamma=1}^{2} \widehat{C}_{0}^{8}(\gamma, 10)+\sum_{\omega=1}^{1} \widehat{C}_{0}^{8}(\omega+1,10) \\
& =\left(\begin{array}{c}
11 \\
3
\end{array}\right)-\left(\begin{array}{l}
9 \\
2
\end{array}\right)-\left(\begin{array}{l}
7 \\
2
\end{array}\right)-\left(\begin{array}{l}
5 \\
2
\end{array}\right)-\left(\begin{array}{l}
3 \\
2
\end{array}\right) \\
& -\left(\begin{array}{l}
6 \\
1
\end{array}\right)-\left(\begin{array}{l}
4 \\
1
\end{array}\right)-\left(\begin{array}{l}
8 \\
1
\end{array}\right)-\left(\begin{array}{l}
6 \\
1
\end{array}\right)-\left(\begin{array}{l}
7 \\
0
\end{array}\right)+\left(\begin{array}{l}
6 \\
0
\end{array}\right)+\left(\begin{array}{l}
4 \\
0
\end{array}\right)+\left(\begin{array}{l}
4 \\
0
\end{array}\right) \\
& =165-36-21-10-3-6-4-8-6-1+1+1+1=73 .
\end{aligned}
$$

Whilst we cannot verify this is the correct value of the Möbius function on this interval, the example serves as a good indicator of the efficiency of the conjecture if it can be proved correct.

\section{Acknowledgements}

I would like to thank Eva Hauksdóttir for suggesting some of the results in the statement of Theorem 5 and my advisor Einar Steingrímsson for many helpful comments. I would also like to express my gratitude to an anonymous referee for extremely detailed, useful and in depth comments, for suggesting the rewriting of the proof of Proposition 4 that is presented here and pointing out an error, now fixed, in Lemma 8. 


\section{References}

[BF13] Mathilde Bouvel and Luca Ferrari. On the enumeration of d-minimal permutations. Discrete Mathematics \&6 Theoretical Computer Science, 15(2), 2013.

[BJJS11] Alexander Burstein, Vít Jelínek, Eva Jelínková, and Einar Steingrímsson. The Möbius function of separable and decomposable permutations. J. Combin. Theory Ser. A, 118(8):2346-2364, 2011.

[ST10] Einar Steingrímsson and Bridget Eileen Tenner. The Möbius function of the permutation pattern poset. J. Comb., 1(1):39-52, 2010.

[Sta12] Richard P. Stanley. Enumerative combinatorics. Volume 1, volume 49 of Cambridge Studies in Advanced Mathematics. Cambridge University Press, Cambridge, second edition, 2012.

[Ste13] Einar Steingrímsson. Some open problems on permutation patterns. Surveys in Combinatorics 2013 (Simon R. Blackburn, Stefanie Gerke and Mark Wildon, eds.) Cambridge University Press, Cambridge, 239-263, 2013.

[SV06] Bruce E. Sagan and Vincent Vatter. The Möbius function of a composition poset. J. Algebraic Combin., 24(2):117-136, 2006.

[Smi14] Jason P Smith. On the shellability and Möbius function of intervals of permutations with a fixed number of descents. In preparation. 